\title{
Mode shift at bottleneck increasing transit dispatch in peak time
}

\author{
ZHONG Lan-fen ${ }^{1,2}$, WANG Wen-zhong ${ }^{2, a}$ \\ ${ }^{1}$ College of Computer Science, Inner Mongolia University, Hohhot 010021, China \\ ${ }^{2}$ Department of Information Science and Technology, Baotou Teachers' College Inner Mongolia University of Science \&Technology, \\ Baotou 014030, China
}

\begin{abstract}
For attracting more auto commuters to shift to transit mode and mitigating congestion at a bottleneck in morning \&rush hour, additional dispatches of transit are operated during peak time. Classical bottleneck model combining with Logit-based discrete choice formula is extended to investigate commuters' mode choice behaviors between private car and public transit. The existence of bi-mode user equilibrium when tolling auto commuter is proofed, and waiting time and time delay costs are formulated in two modes when additional buses are dispatched. Numerical experiments are conducted to examine mode split patterns and aggregate travel cost when additional dispatching service interval varying. Our results show the system aggregate trip cost would reduce prominently when extra buses are added into runs in appropriate time. Especially when waiting time equals 0.65 hour for auto commuters, the system aggregate trip cost would reduce by $70 \%$ in theory.
\end{abstract}

\section{Introduction}

As the development of economy and society, the Traffic Authority (TA) around the world are challenged by using limited road space in green way to provide transportation service in city. For reducing congestion and pollution, various traffic management strategies have been proposed by researchers, such as congestion toll, parking policy, auto restraint, and transit service. Effective transit service is one of empirical ways to reduce congestion and protect environment. The challenge for transit system is to attract people, besides transit captive users, out of cars, especially in congested bottleneck, since that Traffic jam always appears at bottleneck in morning and evening rush hour. One of the adopted strategies is to encourage park-and-ride travel, namely leading auto commuters to park around bottleneck, then they shift to a public transit to go through congested corridor of road and accomplish the rest of trip. More commuters are to switch over to public transport when increasing the frequency of bus dispatch. However, increasing either dispatch frequency or expanding capacity is not the most cost-efficient instrument, especially when commuting is strongly concentrated in peak period, for increasing capacity will be largely idle outside peak period. In the most of the literatures of bi-mode study, transit dispatch frequency is fixed in all service time for management convenience, but realistic commuting demand is varying with time in a day. So, in this paper, what brings forth new idea is we consider to take some additional bus dispatch into routine dispatched transit service in morning rush hour at bottleneck to attract auto commuter to shift to take bus, thereby mitigating congestion at a bottleneck in morning rush hour.

Vickrey proposes one of the most insightful and tractable bottleneck model to analyze morning commuting way at bottleneck [1]. The work on the bottleneck congestion in morning commuting with two modes (car and transit) has also been developed. Tabuchi studies pricing and mode split in a bi-mode competitive system containing transit and high way [2]. Huang extends Tabuchi's study of mode choice by introducing in-vehicle crowding congestion and analyze how to optimize bottleneck capacity utilization by adjusting car congestion toll, transit fare, mode split, and dispatch frequency [3-5]. Kraus studies the optimality of fare, serving number of train unit and capacity of individual train unit [6-7]. Li and Zheng proposes a time-dependent pricing scheme to motivate mode shift and increase the efficiency of pricing to attain equitable savings for all users[8-9]. All these research work reveal the rule of commuting behavior and the performance of tolling

${ }^{a}$ Corresponding author: wwenzhong@126.com 
regimes at bottleneck. But, the effect of additional transit dispatch on decreasing travel cost and the degree of congestion at bottleneck has rarely been considered in these literatures. Our outstanding work is focused on it.

Besides, mode choice between transit and auto is a complex decision process, which is influenced by many factors. To account for these factors in practice, mode split functions have been developed. One of the most early and widely used mode split functions is Logit-based discrete choice formula. Recently, Lu proposes a nested Logit-based stochastic user equilibrium model to characterize the mode choice of commuters and finds that public subway and private parking lot can approximately generate the maximum expected net benefit of transport system while motivating transit travel[10]. More recently, Kitthamkesorn develops a Logit-based model to alternate the weibit-based model for the combined modal split and traffic assignment problem, which is effective to quantitatively evaluate and examine the behavioral modeling of travelers' mode shift between private motorized mode and go-green modes [11]. From lane perspective, Logit formula is applied to investigate two types mode split problems: physically separated rail and together rail. When the rail of train or subway is viewed as parallel lane with auto road, $\mathrm{Lu}$ and Kitthamkesorn's results are suitable to analyze competitive bus and auto traffic system with dedicated bus lane. About dedicated bus lane strategies, please refers to existing literatures [12]. Here, it is assumed that buses take its own lane and what we concern is how dispatch additional buses to find a system cost optimum solution.

Gonzales and Daganzo provided a system optimum solution with two modes, and presented pricing method to achieve the system optimum in the condition that commuters arrive and pass a bottleneck in order of wished departure [13]. The study has made progress in competitive transportation system modeling in peak time; nevertheless, its mode split, pricing and system optimum are based on the uses equilibrium which is derived from the principle that transit user and auto user have the same cost in equilibrium, which is impractical for all cases, since mode choice results from a complex decision process, not following user equilibrium principle. we will further the bi-mode equilibrium study with Logit-based formula, meanwhile system user optimum cost will be discussed when additional transit service starts to be provided at a certain time point.

The paper is organized as follows. Section 2 formulates the equilibrium commuting patterns of two modes. Section 3 analyzes the travel costs of both private auto and public transit at equilibrium. Section 4 gives numerical results for intuitively demonstrating the mode split and commuting patterns with respect to some different input parameters. Section 5 concludes the paper.
In the bottleneck model, $N$ commuters have to go through a bottleneck from $O$ (home place) to $D$ (work place) in morning rush hour. The bottleneck's capacity is fixed at $S$. If arrivals at the bottleneck ever occur at a rate exceeding $S$, then a queue develops. Here all commuters are assumed to be identical in perceiving time value, and congestion would happen at bottleneck only.

When only auto mode is used, there exists a highly congested duration around $\left(t_{1}, t_{2}\right)$, as shown in Figure 1. When an alternative public transit service becomes available, from simple geometrical considerations, the transit service operation aims to shift the portion of commuters represented by the area of triangle BCD (shown in Figure.1.) from auto mode to transit mode. It is assumed in this section that the TA charges a fixed fare and operates buses at a fixed headway. There are two kinds of user by transit. One is captive user, and the other is choice user. For simplifying problem, we assume captive transit users can be served by routine transit dispatches. Here only considers the choice users who can alternate two modes. The transit service mentioned following refers to the additional dispatched buses besides routine transit buses. So we make three assumptions. (i) Commuters are choice users, who can change their travel mode arbitrarily. (ii) Routine transit is available in whole service time, and additional transit service is only available during highly congested period of rush hour. (iii)When transit is operating, it is fully segregated on its own lane, so that transit service is not subject to traffic congestion. All choice travelers adjust their departure time and travel mode to reduce their travel cost. An equilibrium is obtained when no individual has an incentive to change his departure time and travel mode. Actually, two types of equilibrium arise at the bottleneck: one is experienced by auto commuters, and the other is mode split pattern which means the ratio of auto commuters to bus commuters is invariable when equilibrium state comes up.

In Figure $1, t^{*}$ denotes the work starting time. Without restriction, the free flow travel time form $O$ to $D$ is assumed to be zero. Let $\tilde{t}$ be arrival time at bottleneck for punctuality for work. So commuters arriving at bottleneck before $\tilde{t}$ are early, and commuters arriving at bottleneck after $\tilde{t}$ are late. Each unit time of earliness is associated

\section{User equilibrium}


with a penalty of $\beta$ and each unit time of lateness is

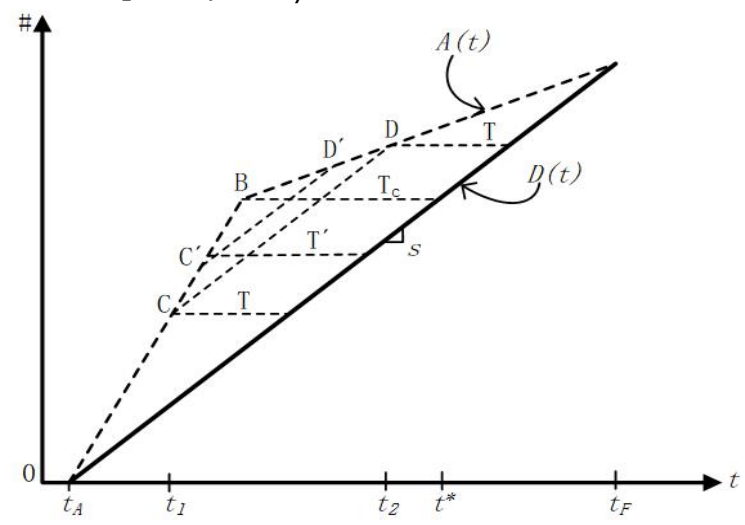

Figure 1.User equilibrium for a fixed capacity bottleneck in single auto mod

$\gamma \cdot \alpha$ is waiting time cost rate. The slope of the arrival curve in equilibrium, shown in Figure 1, satisfies:

$$
A^{\prime}(t)= \begin{cases}s /(1-\beta / \alpha), & t \in\left[t_{A}, \tilde{t}\right), \\ s /(1+\gamma / \alpha), & t \in\left(\tilde{t}, t_{F}\right] .\end{cases}
$$

The queuing time increases at rate $\beta /(\alpha s)$ with each additional commuter in the time interval $\left[t_{A}, \tilde{t}\right)$ and the queuing time declines at the rate $\gamma /(\alpha s)$ with each departing commuter in $\left(\tilde{t}, t_{F}\right]$. Let $W(t)$ denote the waiting time when a commuter who arrives at bottleneck at time $\mathrm{t}$ would experience. $W^{\prime}(\mathrm{t})$ representing waiting time rate, meets:

$$
W^{\prime}(\mathrm{t})= \begin{cases}1 /(\alpha / \beta-1), & t \in\left[t_{A}, \tilde{t}\right), \\ 1 /(\alpha / \gamma+1), & t \in\left(\tilde{t}, t_{F}\right]\end{cases}
$$

$D(t)$ denotes cumulative arrivals at the bottleneck at time $\mathrm{t}$ and $D^{\prime}(t)=s$. When only auto mode is used, in equilibrium, a critical commuter with departure time $\tilde{t}$ arrives at work on time but experiences the maximum waiting time $T_{c}=\beta \gamma N /(\beta+\gamma) \alpha s$ as a queuing delay, and $\tilde{t}=t^{*}-T_{c}$.

The waiting time for commuters who arrival at $t_{1}$ or $t_{2}$ (shown in Figure 1) is the same, and it is assumed as $T$.

From preceding assumptions, if the transit service is provided during $\left[t_{1}, t_{2}\right]$, there must have a certain number of commuters who will take it, and the waiting time $W(t)$ for any auto commuter, would be reduced. When bi-mode transport system gets its equilibrium, $W(t)$ is supposed to be equal to $T$. However, it follows that other commuters will choose to pass the bottleneck in this time interval due to the decrease of the waiting time, which in turn results in $W(t)$ increased. Therefore, a toll varying wite time $t$ should be implemented during $\left[t_{1}, t_{2}\right]$. Toll, denoted by $\tau(\mathrm{t})$, satisfies: satisfies:

$$
\tau(\mathrm{t})=\left\{\begin{array}{l}
\beta\left(t-t_{1}\right), t \dot{o}\left[t_{1}, \tilde{t}\right), \\
\gamma\left(t_{2}-t\right), t \dot{o}\left(\tilde{t}, t_{2}\right] .
\end{array}\right.
$$

Proof. In equilibrium, the auto commuters departing to join in the bottleneck experience the same waiting time $T$, which means $A^{\prime}(t)=0, t \in\left[t_{1}, t_{2}\right]$. The amount of toll is corresponding to the amount of cost which is lessened on account of available additional transit service Knowing from formulate (1), the number of queuing commuters increases at $s /(1-\beta / \alpha)$ per unit time, and the queuing time increases at rate $\beta / \alpha s$ with each additional commuter in the time interval $\left[t_{1}, \tilde{t}\right)$, so in per unit time the queuing time for auto commuter increases at $s /(1+\gamma / \alpha)$. Toll should balance the sum of decreased queuing time cost and increased early schedule delay cost. Therefore, $\tau(\mathrm{t})=\left(\alpha \beta /(\alpha-\beta)-\beta^{2} /(\alpha-\beta)\right)\left(t-t_{1}\right)=\beta\left(t-t_{1}\right)$. Likewise, when $t \dot{o}\left(\tilde{t}, t_{2}\right]$, we can get $\tau(\mathrm{t})=\gamma\left(t_{2}-t\right)$.

At equilibrium the mode split at aggregate demand level is governed by a Logit formula specified below

$$
N_{t}=N /\left(1+\exp \left(\theta C_{t}-\theta C_{a}\right)\right) .
$$

Where $\theta$ is a positive parameter relating to the standard deviation of random variables and its value can be estimated from survey data; and $C_{a}$ and $C_{t}$ are trip cost for each auto and transit commuter respectively. $N_{t}$ and $N_{a}$ are the distributing number of commuters for mode transit and auto respectively. And $N=N_{t}+N_{a}$.

When more bus runs are dispatched in peak time, some auto commuters are supposed to be attracted to take bus. An equilibrium is easy to find when the mode split is governed by Logit formula (4).Here, it is assumed that the population choosing to take bus, represented by $N_{t}$, is geometrically shown by the triangle area BCD in Figure 1, and it follows that the maximum queuing time for auto commuter related to $N_{a}$ is $T$.

For demonstrating commuters no intensive to change their mode, we consider the trip cost when transit service is provided on a later time, $t_{1}^{\prime}$ (corresponding to $C^{\prime}$ ), as shown in Figure 1. According to no-toll equilibrium, presented by Arront [14-17], the quantity of $T$ does not change the trip cost of auto user who arrives at the bottleneck when the transit operating begins. So it is intuitive to know $C\left(t_{1}^{\prime}\right)=C\left(t_{1}\right)$. But when transit service and the implementation of time-dependent toll rule actually starts at time $t_{1}$, the trip cost $C\left(t_{1}^{\prime}\right)$ becomes larger. That is $C\left(t_{1}^{\prime}\right)=C\left(t_{1}\right)+\tau\left(\mathrm{t}_{1}^{\prime}\right), \tau\left(\mathrm{t}_{1}^{\prime}\right)>0$. i.e. $C\left(t_{1}^{\prime}\right)>C\left(t_{1}\right)$, so commuters have no intensive to change their mode split situation, and here comes equilibrium state. 


\section{Travel costs}

For determining equilibrium mode split pattern, it suffices to figure out auto traveler individual cost and transit user individual cost, respectively.

\subsection{Auto mode}

Individual auto commuter's total travel cost(ATTC) depends on waiting time, schedule delay, toll and vehicle expenses (e.g., fuel consumption and vehicle depreciation). To simplify, we assume a linear travel cost function:

$$
C_{a}(t)=\alpha T_{w}(t)+d(t)+\tau(t)+\eta_{1} .
$$

Where $C_{a}(t)$ is ATTC who arrives at the bottleneck at time $t, T_{w}(t)$ is the waiting time in the queue, $d(t)$ is the schedule delay cost, $\tau(t)$ is toll, and $\eta_{1}$ represents vehicle expenses. In the equilibrium, any auto commuter is unable to find an arrival time at the bottleneck) which can reduce ATTC. In other words, $\mathrm{d} C_{a}(t) / d t=0$. Hence, all they have the same ATTC, $C_{a}$. The first and last auto commuter of arrival at bottleneck in the time interval $\left[t_{1}, t_{2}\right]$, face the same waiting time, $T$, and have the same travel cost $C_{a}$, i.e.,

$$
\begin{aligned}
& C_{a}\left(t_{1}\right)=C_{a}=\alpha T+\beta\left(t^{*}-t_{1}-T\right)+\eta_{1} . \\
& C_{a}\left(t_{2}\right)=C_{a}=\alpha T+\gamma\left(t_{2}-t^{*}+T\right)+\eta_{1} .
\end{aligned}
$$

During $\left[t_{1}, t_{2}\right]$, the numbers of cars going through the bottleneck are $N_{a}$, and we then have

$$
N_{a}=s\left(t_{2}-t_{1}\right)
$$

Combining equation (6) and (7), we can determine the following times unknown:

$$
\begin{gathered}
t_{1}=t^{*}-T-\gamma N_{a} /(\gamma+\beta) s \\
t_{2}=t^{*}-T+\beta N_{a} /(\gamma+\beta) s .
\end{gathered}
$$

Substituting (10) and (11) into (7) and (8), respectively, $C_{a}$ can be expressed as:

$$
C_{a}=\alpha T+\beta \gamma N_{a} /(\gamma+\beta) s+\eta_{1} .
$$

Furthermore, when $t_{1}$ and $t_{2}$ are determined, we can get $t_{A}$ and $t_{F}$ using the below expressions.

$$
\begin{aligned}
& t_{A}=t_{1}-(\alpha-\beta) T / \alpha s^{2} . \\
& t_{F}=t_{2}+(\alpha+\gamma) T / \alpha s^{2} .
\end{aligned}
$$

\subsection{Transit mode}

Now, we consider the travel cost happens on commuters who select dispatched buses as their commuting mode during $\left[t_{1}, t_{2}\right]$. The cost should depend on waiting time at original bus stop, schedule delay, bus fare and discomfort generated by body congestion in bus carriage. Then, individual transit total travel cost (TTTC) at time $\mathrm{t}$ is

$$
C_{t}(t)=\alpha T_{w}(\mathrm{t})+d(\mathrm{t})+f(\mu)+\eta_{2} .
$$

$T_{w}(\mathrm{t})$ is waiting time at origin bus stop, $d(\mathrm{t})$ is schedule delay cost, $f(\mu)$ is body congestion in bus carriage with $\mu$ capacity limit, and $\eta_{2}$ is bus fare. From transit commuter costumer view, the average cost is crucial for the determination of mode choose. So here we use $C_{t}$ represents the average trip cost for a transit user.

$$
C_{t}=\alpha T_{w}+\delta+f(\mu)+\eta_{2} .
$$

$T_{w}, \delta$ is waiting time at the original stop and schedule delay cost respectively in average. Each transit run has a strict capacity of $\mu$, the number of runs is denoted by $R$, $R=N_{2} / \mu$, and the number of physically distinct buses used to make the runs is denoted by $K$. The time length for a bus to make an around trip is $d$ minutes. Following the optimal schedule of running buses in clusters [7], we expend the clusters from before $t^{*}$ to the whole peak time. From what has been discussed above, for any given $T$, in equilibrium, $C_{a}$ is the function of the number of commuters $N_{a} ; C_{t}$ is determined by $N_{t}$ in term of given $\{\sigma, \mathrm{K}, \partial, d\}$. Then $C_{a}$ and $C_{t}$ can be written as $C_{a}=C_{a}\left(N_{a}\right) \quad C_{t}=C_{t}\left(N_{t}\right)$.Using the logit formula described in Section 2, $N_{1}$ and $N_{2}$, the split pattern, can be determined under the condition of given $T$. Note that the problem of finding a mode split is in fact to solve a fixed point problem because the equilibrium travel costs $C_{a}$ and $C_{t}$ depend on $N_{a}$ and $N_{t}$, respectively. Then passenger flows references, $t_{1}, t_{2}, t_{A}$, and $t_{F}$, can be determined by the equations (8)- (12).

\section{Numerical results}

The input parameters of our numerical example are: $N=5000$ (persons), $(\alpha, \beta, \gamma)=(6.2,3.7,15.2)(\$ / h)$ $, t^{*}=8: 00 \mathrm{Am} \quad, s=2500(\operatorname{cars} / h), \partial=5(\mathrm{mins})$, $\eta_{1}=14(\$ /$ trip $), \eta_{2}=2(\$ /$ person $), \theta=0.05$. We assume $d=45$ (minutes) $\quad, \quad K=8$ (units)

$f(\mu)=-5 \ln (1-\sqrt[4]{\mu / 80})$. When $T$ equals 0.5 hours, we got $\left(N_{1}, N_{2}\right)=(1351,1071)$.Figure 2 depicts the number of commuters using auto mode and mode split patterns when $T$ varies from 0 to $T_{c}$, respectively. Here, $T=0 \mathrm{~h}$ means transit service is available in all morning rush hour, and the rate of auto users to transit users is 3 to 2 . As waiting time $T$ increases, the two-mode period decreases and the rate gradually approximates to 1 to 1 . The number 
of transit users is decreasing because of shorter and shorter service period $t_{2}-t_{1}$. Finally, when $\mathrm{T}$ is approaching 1 , both $N_{1}$ and $N_{2}$ are approaching zero. Figure 2 shows aggregate trip cost of commuters is different when transit service is operated in different waiting time $T$.

The aggregate trip cost of commuters without transit service is $3.1 \times 10^{4}$, while it decreases when transit is available. It can be seen that a local minimum for the aggregate trip cost is $0.9 \times 10^{4}$ at bottleneck if transit is provided when waiting time $T$ equals $0.65 \mathrm{~h}$, shown in Figure 3. The aggregate trip cost drops by $2.2 \times 10^{4}$ theoretically, about $70 \%$ cost saving.

Besides, compared with the data of the numerical experiment in Huang [5] literature, the aggregate trip cost in our experiment is roughly less than the Huang's .

Our dispatching headway is 5 minutes, but Huang's is 2 min; and our model's peak duration becomes shorter duo to additional dispatches. It means more buses are needed in Huang's model than our model dose, and the more running buses inevitably cause the more heavily environmental pollution and the more transit agency cost.

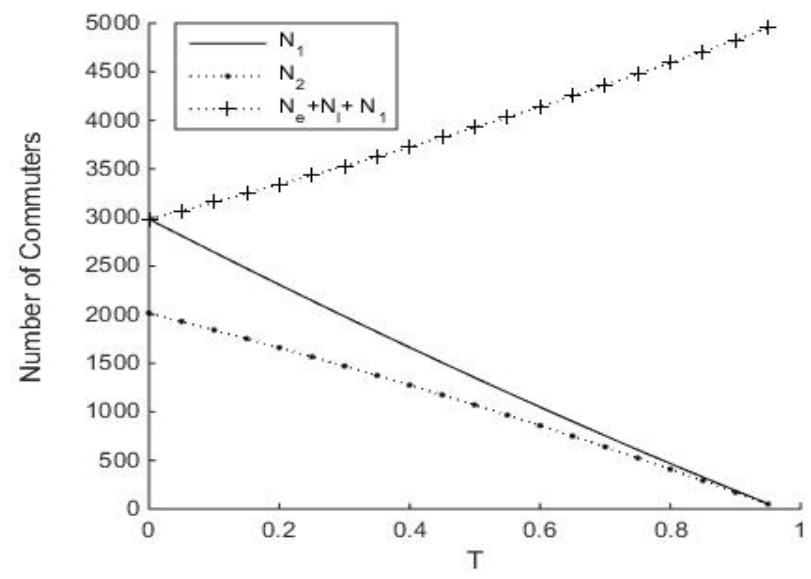

Figure 2.The mode split patterns when $T$ varying

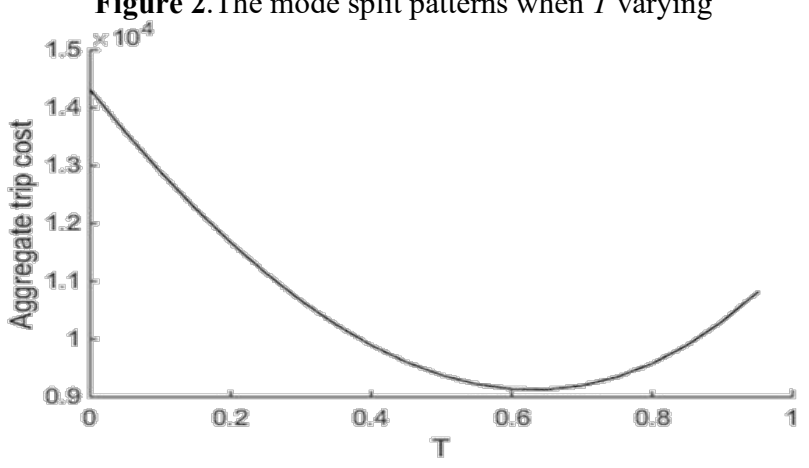

Figure 3. The aggregate trip cost of commuters varies with $T$

\section{Conclusion}

In this paper we study the mode choice and commuting behaviors at a bottleneck with a bi-mode transportation system based on Vickery's bottleneck model and Kraus's study when an additional transit service is provided in rush hour. The bottleneck model are developed to depict peoples commuting behaviors by auto and transit modes, respectively. We develop Kraus's qualitative results to quantitative experimental conclusions. The mode choices is governed by a logit-based model. We analytically and numerically investigate the properties of equilibrium state and the consequences of trip cost by providing additional transit service at different time duration.

Through this study, we give new insights into peoples' mode choice and aggregate trip cost at the bottleneck with time-constrained transit service. The main results gained can be summarized below: (i) Everyone experiences a lower cost when additional transit service is provided than if it is not. Theoretically more than half of aggregate commuter trip cost is saved. (ii) Commuters aggregate trip cost gets its optimum value when additional transit service begins when waiting time is a half of the maximum waiting time $T_{c}$, i.e. $T=T_{c} / 2$. Looking at our findings, two stand out. One is that dispatching additional buses during the peak time of rush hour is an effective way to reduce congestion at bottleneck, which can save commuters aggregate trip cost a lot. The second is that with numerical experiment help we find out the optimum service duration of additional dispatches, which has practical value in reality.

The above results rely on our analyses carried out in a simple bi-mode, exclusive bus lane transportation system. Hence, the generality of the results is restricted to some extent. For simplicity, we assume that all commuters are homogeneous choice users. The modeling of multiple modes can be further improved by considering additional runs among pre-existing runs serving captive bus users. For example, only one part of bus passenger capacity remains for choice users, and the other part is default for captive users.

\section{Acknowledgments}

This project has received funding from National Natural Science foundation of China (grant agreement 71622005) http://www.nsfc.gov.cn.

\section{References}

1. W S Vickrey. AER, J.59 (2) (1969)

2. T. Tabuchi. J URBAN ECON, J.34 (3) (1993)

3. H J Huang. TRANSPORT RES E, J. 36(4) (2000)

4. H J Huang. EUR J OPER RES, J. 140(3) (2002).

5. H J Huang, et al. TRANSPORT RES E, J.43(5) (2007)

6. M Kraus, Y Yoshida. J URBAN ECON, J. 51(1) (2002)

7. Marvin Kraus. J URBAN ECON, J. 4(3) (2003)

8. Li S. Prompt-Traffic \& Transportation, J. 24(5) (2012)

9. N Zheng, et al. TRANSPORT RES, J. 62(2) (2016)

10. X S Lu et al. Transport Policy. 44 (2015)

11. S Kitth, A Chen. TRANSPORT RES C, J. (2007)

12. Bagloee et al.Public Transport, 1-29(2017)

13. E J Gonzales, C F Daganzo. TRANSPORT RES B

J. 46(10) (2012) 
14. R Arnott, A D Palma. J URBAN ECON, J.27 (1) (1990)

15. R Arnott, et al. REG SCI URBAN ECON, J.22 (1) (1992)
16. R Arnott. Boston College Working Papers in Economics. 28(2)(1993).

17. R Arnott, A Yan. RURDS, J. 12(3) (2000) 